\title{
Tracking stations alignment with Kalman tracks at LHCb.
}

Louis Nicolas $^{\mathrm{a}}$, Adlène Hicheur ${ }^{\mathrm{a}}$, Matt Needham ${ }^{\mathrm{a}}$, Jan Amoraal $^{\mathrm{b}}$, Wouter Hulsbergen ${ }^{\mathrm{b}}$, Gerhard Raven ${ }^{\mathrm{b}}$

${ }^{a}$ EPFL / Lausanne, ${ }^{b}$ NIKHEF / Amsterdam

Software for Detectors

NSS - IEEE - Dresden

October 21, 2008 
- The LHCb detector and its tracking system.

- The alignment procedure and its maths.

- Alignment scenario using MC magnet-on data.

- A first glimpse at real data at LHC.

- Conclusions. 
Overview of the Large Hadron Collider beauty detector:

- Forward arm spectrometer operated at the Large Hadron Collider.

- Luminosity of $2 \times 10^{32} \mathrm{~cm}^{-2} \mathrm{~s}^{-1}$.

- $10^{12} \mathrm{~b} \bar{b}$ pairs / year in acceptance, b-production peaked in forward direction.

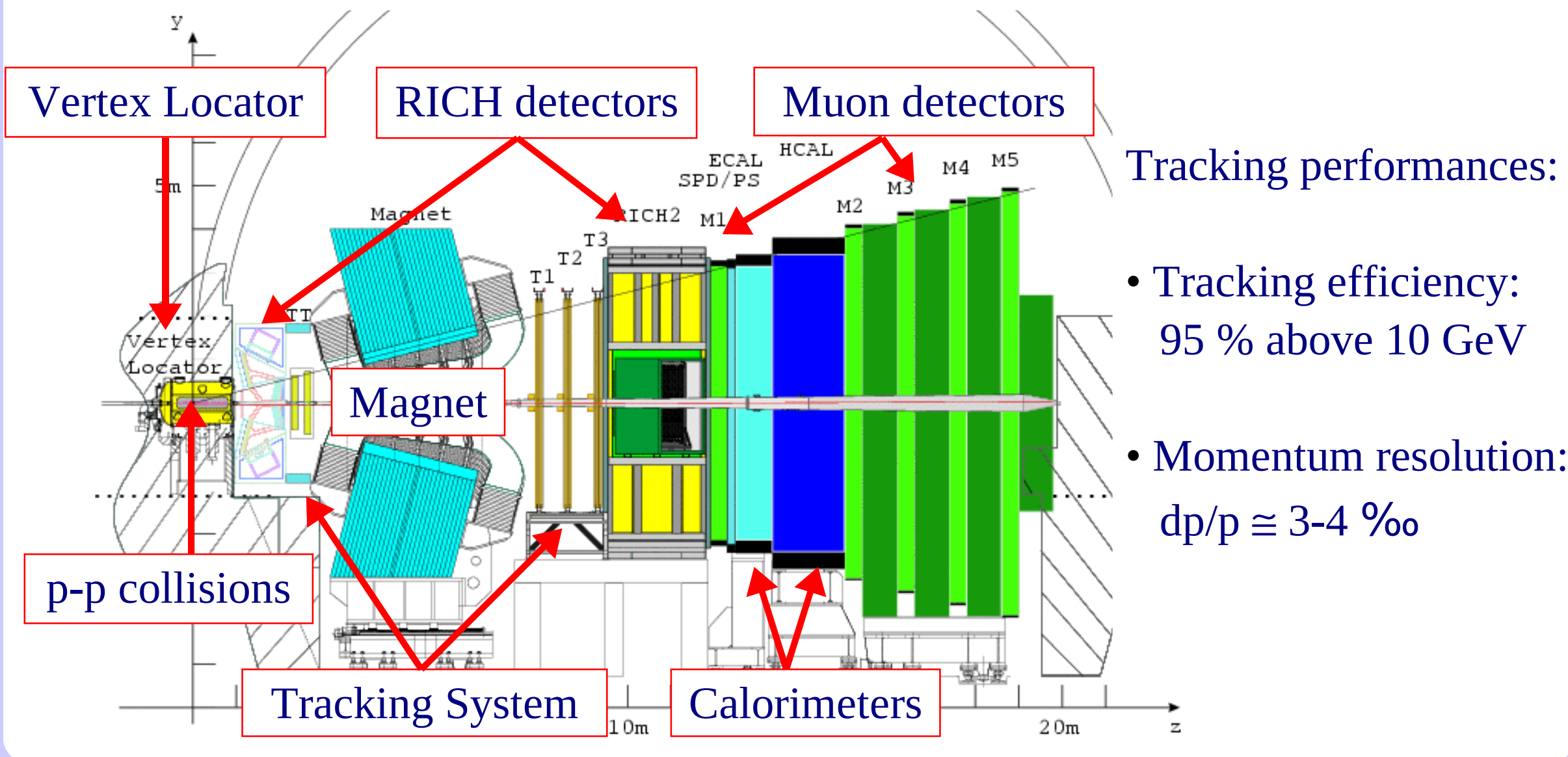


The LHCb tracking system is composed of:

- Trigger Tracker: large silicon detector before magnet.

- Tracking stations after magnet:

- Outer Tracker: straw tubes covering all the acceptance except for the innermost region.

- Inner Tracker: silicon strips detector in region closest to beam pipe.

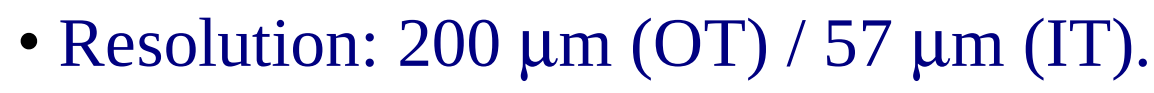

- Hadronic environment, need high tracking efficiency.
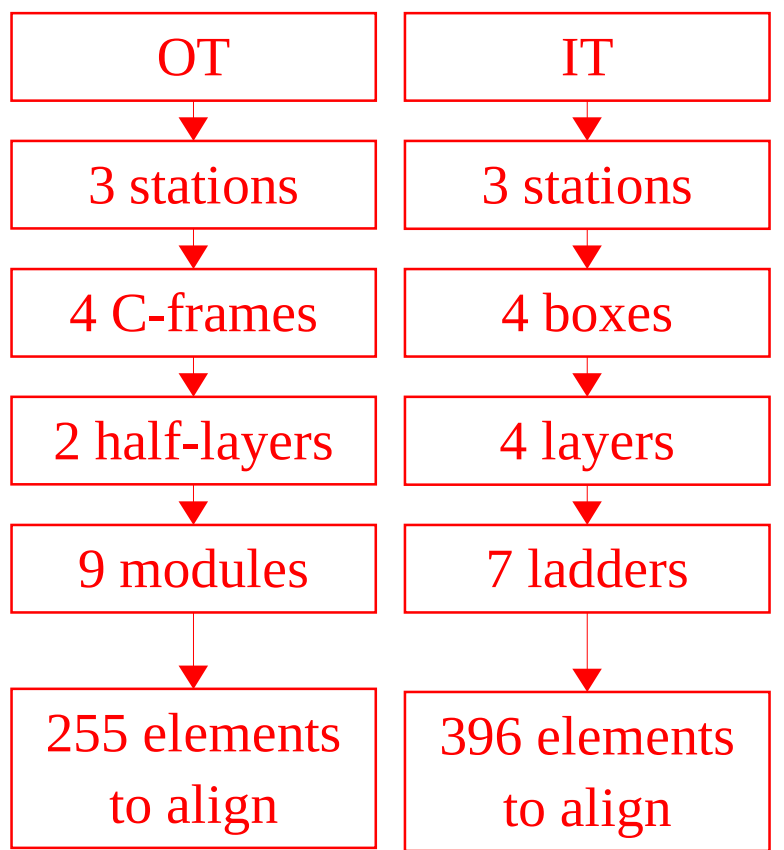

7 ladders

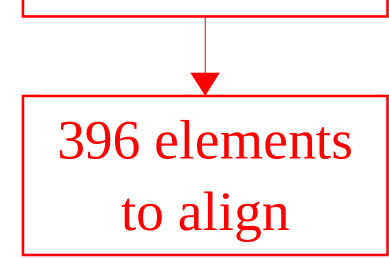

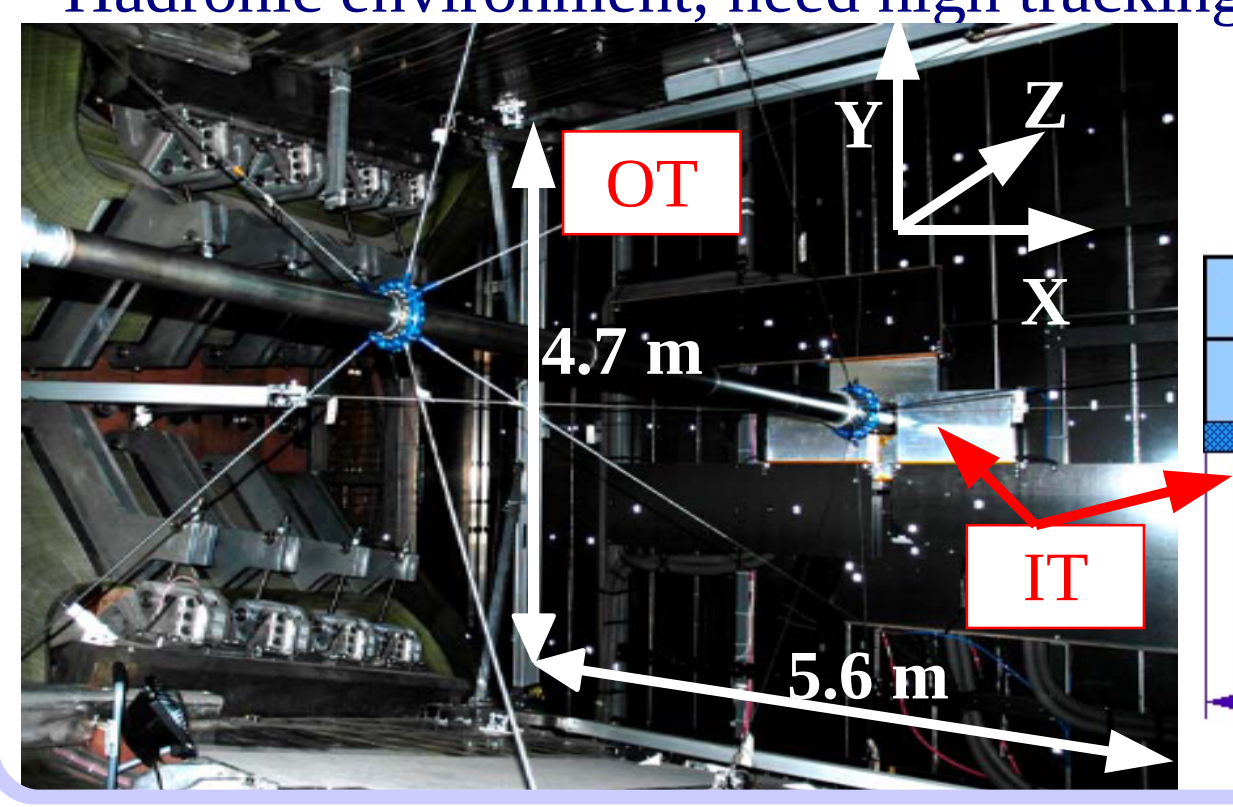

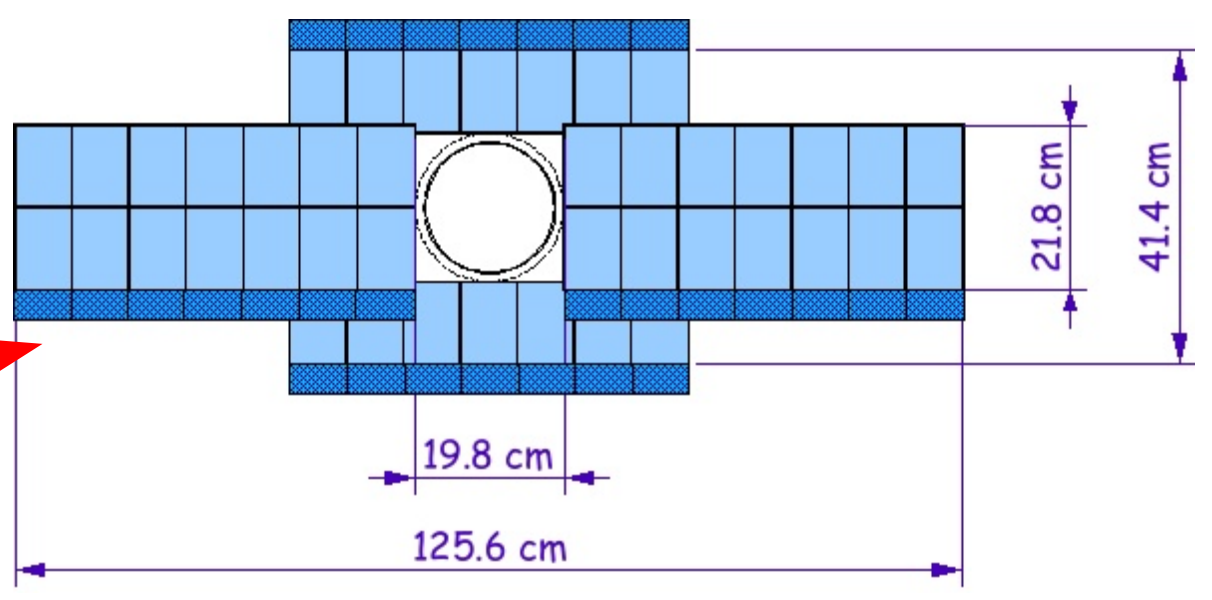


- We use a global $\chi^{2}$ minimisation (Millepede-like).

- V. Blobel, "Software alignment for tracking detectors", NIM A 566 (2006) 5.

- P. Brückman, A. Hicheur and S. J. Haywood, "Global $\chi^{2}$ approach to the alignment of the ATLAS silicon tracking detectors", ATL-INDET-PUB-2005-002.

- A. Bocci and W. Hulsbergen, "TRT alignment for SR1 cosmics and beyond", ATL-COM-INDET-2007-011.

- BUT: we use tracks from the standard Kalman track fit in LHCb. Advantages:

- Use same model for calibration and physics.

- Most complicated model: includes multiple scattering, $\mathrm{dE} / \mathrm{dx}$ correction, ...

- Problem: in calculation, we need global track covariance matrix. This is not given by Kalman track fit, but it can be calculated afterwards. Details of this novel calculation can be found in:

- W. Hulsbergen, "The global covariance matrix of tracks fitted with a Kalman filter and an application in detector alignment", e-Print: arXiv:0810.2241v1 [physics.ins-det] 13 Oct 2008. 
- Use of standard LHCb track fit, magnetic field and geometry framework:

- No global track model needed.

- Possibility to use any standard tracking tool.

- Alignment software: core algorithm using a set of tools, e.g. solving final stage in which regularisation, eigenvalues analysis, etc. can be done.

- Align any detector element. All elements can be aligned simultaneously using long tracks: coherent approach!

- Align for any degrees of freedom: 3 translations + 3 rotations.

- Individual degrees of freedom are selected (e.g. translation along 'y' for TT/IT/OT modules are dropped).

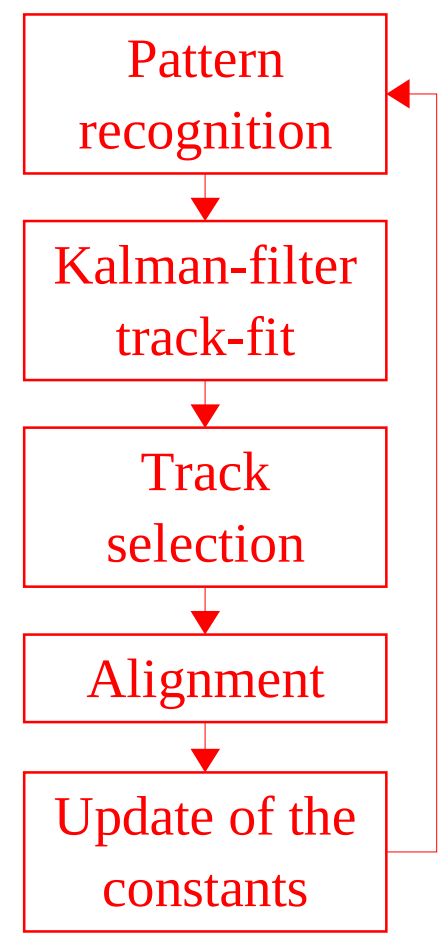

- Iterations of pattern recognition and track fit to solve non-linear system. 
- Solving the alignment equation leads to a big alignment matrix.

- Without any constraints, the matrix is positive definite $==>$ diagonalisable.

- Small eigenvalues correspond to weak modes (large displacements) of alignment.
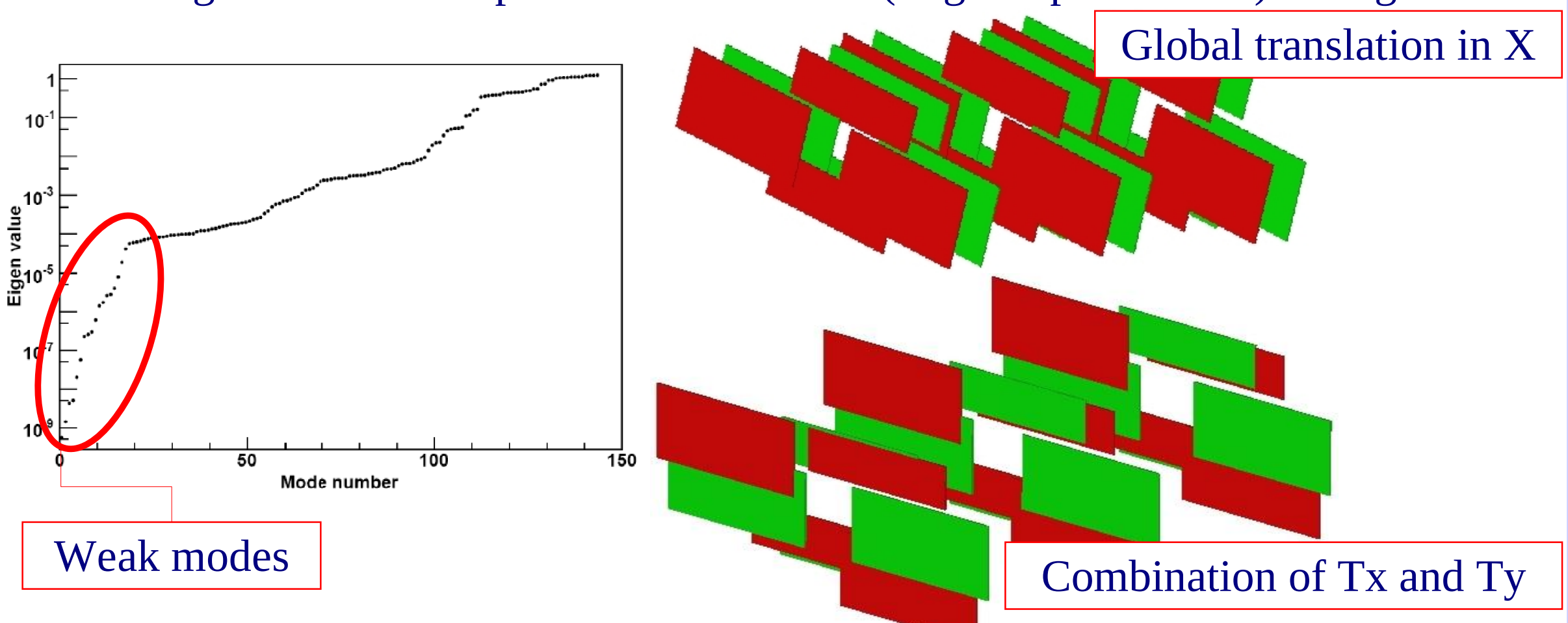

- Constraints can be applied to get rid of weak modes:

- Unconstrained DoFs are constrained with Lagrange multipliers.

- Vertex constraints: information propagated to residuals, correlations computed.

- Cut on eigenvalues to remove unwanted weak modes. 
- Run 8 iterations over 50'000 minimum bias, magnet on events with $7 \mathrm{TeV}$ protons.

- Misalignment scenario:

\begin{tabular}{|ccc|c|}
\hline Detector & Element & DoF & Amplitude \\
\hline \multirow{3}{*}{ IT } & Boxes & Tx $[\mathrm{mm}]$ & 1 \\
\cline { 2 - 3 } & Layers & $\mathrm{Tx}[\mathrm{mm}]$ & 0.1 \\
\cline { 2 - 4 } & Ladders & $\mathrm{Tx}[\mathrm{mm}]$ & 0.05 \\
\hline \multirow{2}{*}{ OT } & \multirow{2}{*}{ Layers } & $\mathrm{Tx}[\mathrm{mm}]$ & 1 \\
\cline { 3 - 4 } & & $\mathrm{Rz}[\mu \mathrm{rad}]$ & 150 \\
\hline
\end{tabular}

- Alignment of IT and OT constrained to VeLo ideal position (using long tracks). Additional constraints: Weak degrees of freedom not aligned for.

- Important points for the convergence of the alignment algorithm:

- Track selection: ==> 1.5 long tracks / event left for the alignment procedure.

- Select events with low occupancy to reduce ghost rate.

- Use evolving $\chi^{2}$ cut to remove ghost tracks and hadronic interactions.

- Multistep approach:

- Align first IT boxes, then IT layers (independent of OT).

- Align OT layers (independent of IT).

- Align IT+OT layers, then IT ladders + OT layers together. 
- $\chi^{2}$ convergence plot for the $5^{\text {th }}$ step (IT ladders + OT layers alignment):

Convergence in 3 iterations.

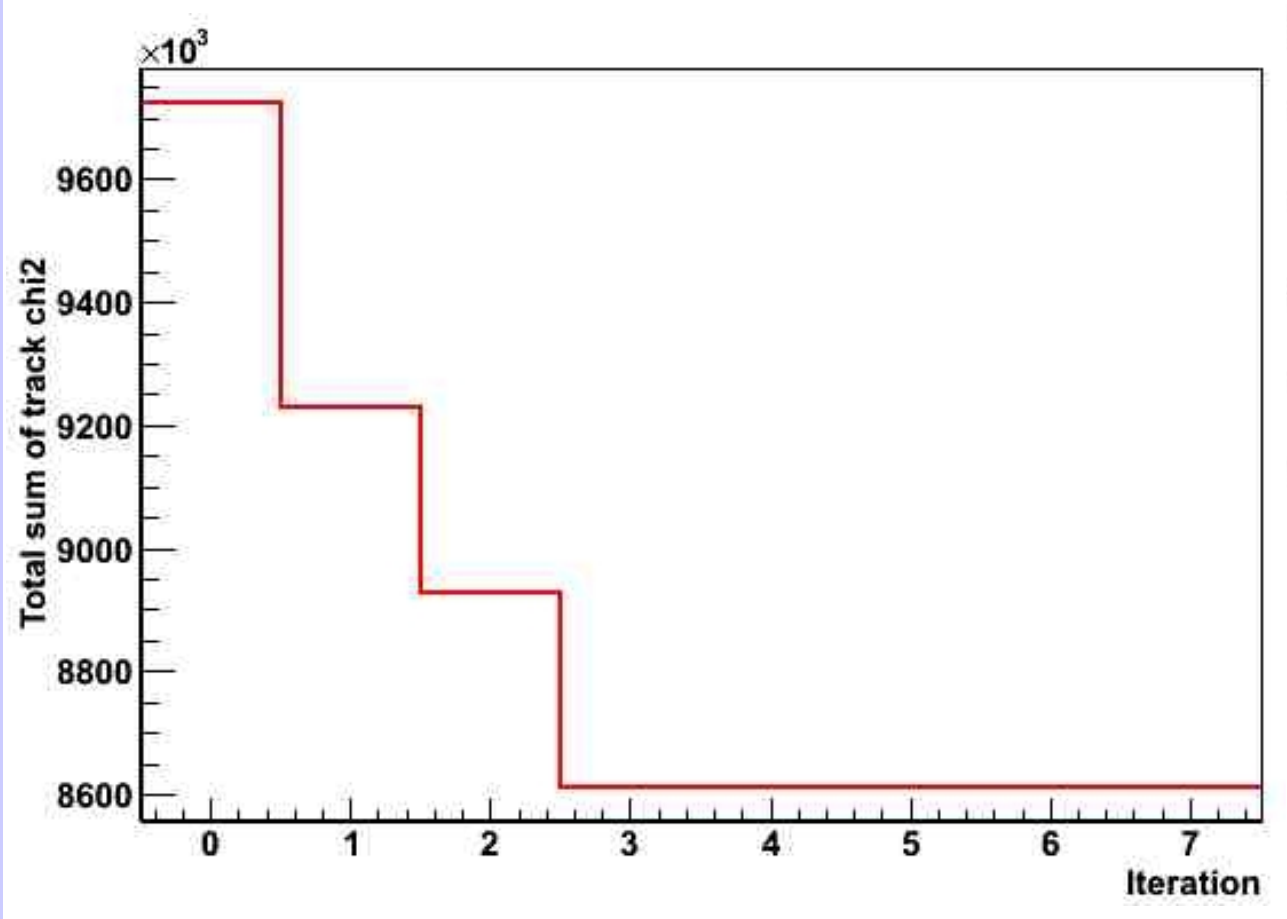

\# hits / element ranges between:

1'000 and 10'000
- Alignment resolution for IT ladders:

(distribution of residual misalignment)

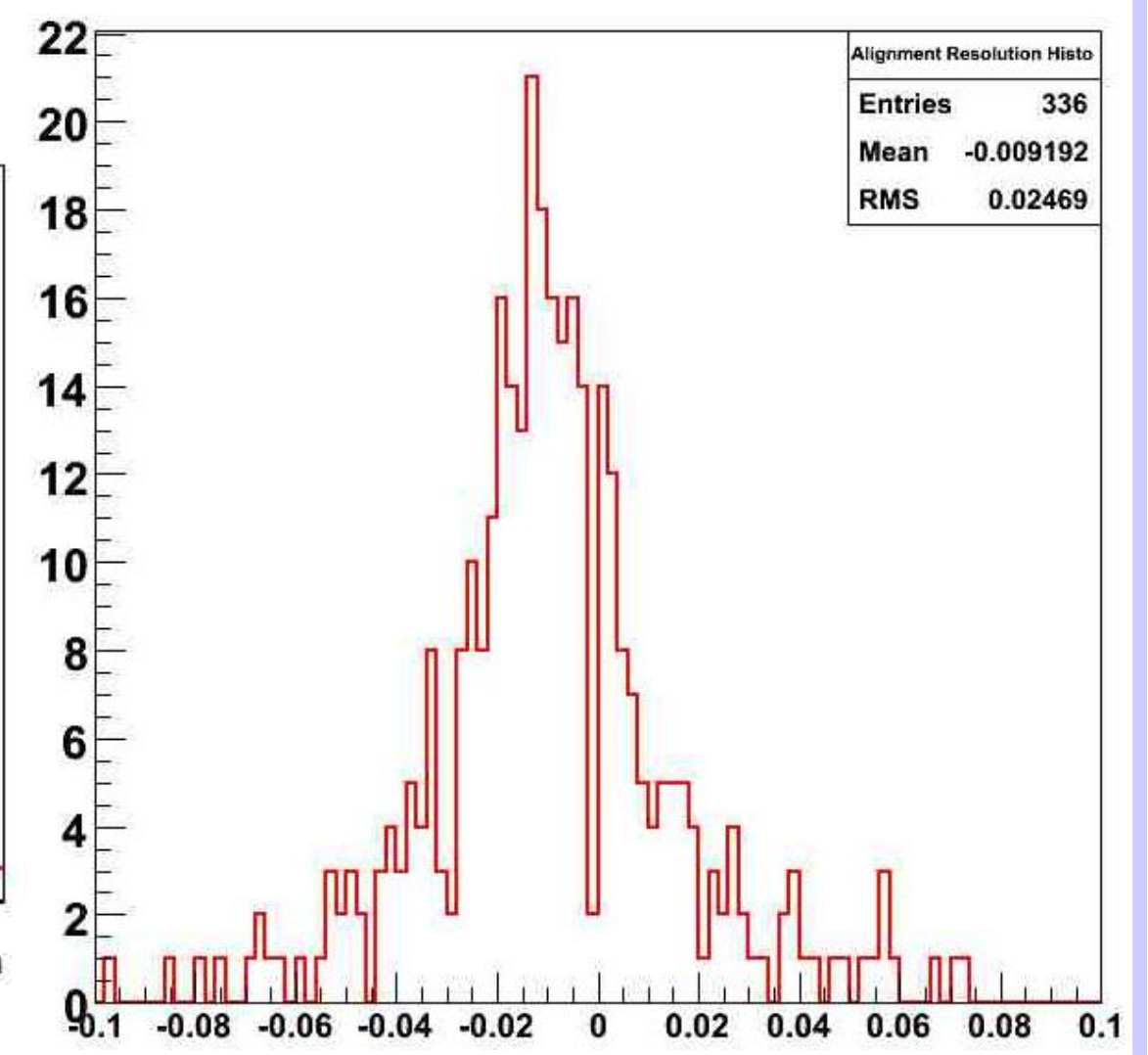

$$
\begin{aligned}
& \mathrm{FWHM}=30 \mu \mathrm{m}==>\sigma \cong 13 \mu \mathrm{m} \\
& \mathrm{Bias} \cong 10 \mu \mathrm{m}(==>\text { bad tracks })
\end{aligned}
$$


Alignment algorithm has converged, but where are we from the physics point of view?

- Run over 65'500 inclusive J/\%.

- Loose $\mathrm{J} / \psi$ selection

$==>31 ' 918 \mathrm{~J} / \psi$ candidates.

- Refit of tracks from $\mathrm{J} / \psi$ with 3 geometries:

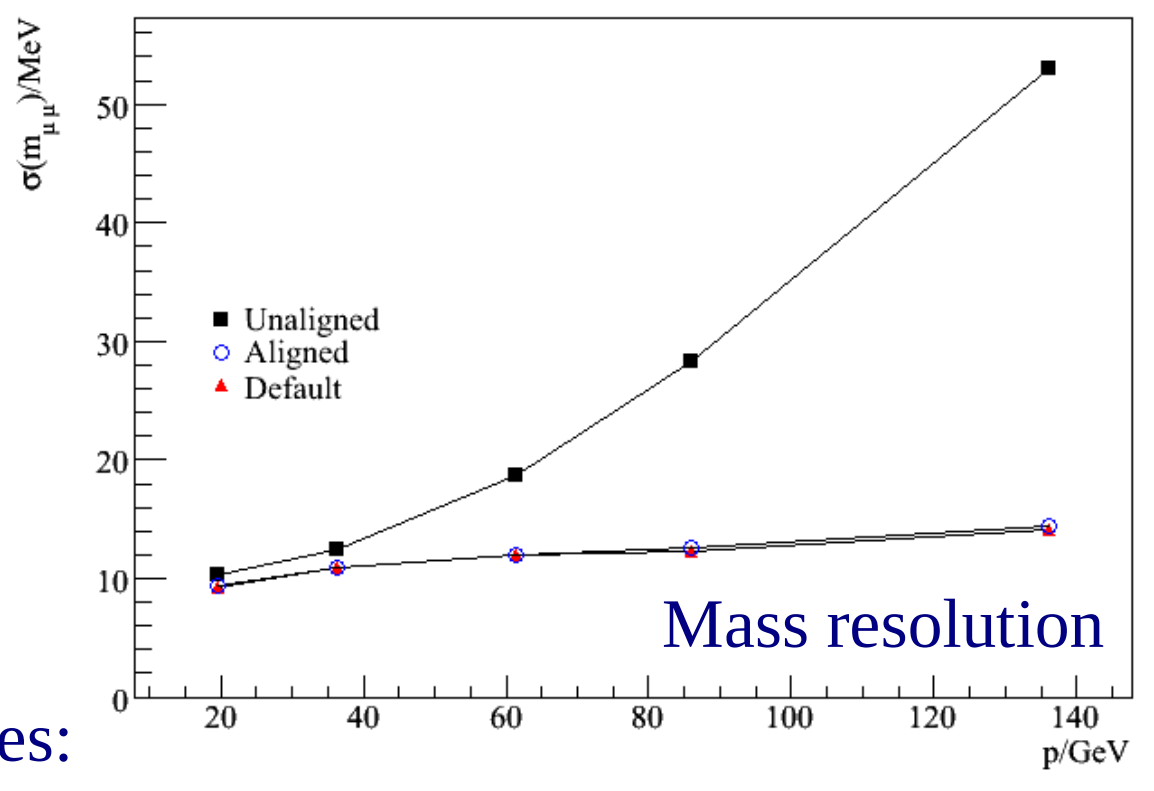

- Unaligned (before alignment job).

- Aligned (after alignment job).

- Default (ideal geometry).

- Look at:

- Dimuon mass resolution:

$$
\text { M(rec) - M(true } \mu \mu)
$$

- Track $\chi^{2} /$ dof.

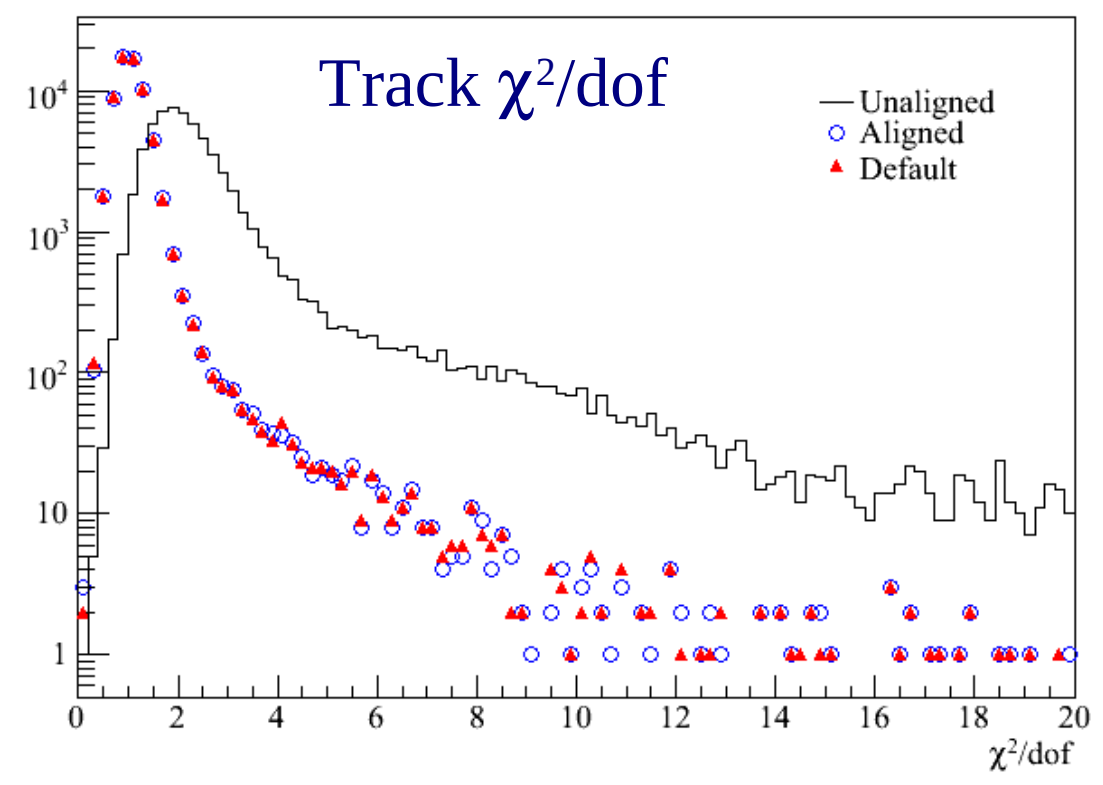


- Data-taking runs during the summer:

- Cosmic rays data: many tracks for OT, but barely any for IT.

- Beam-dump data: very crowded, good for VeLo but not for tracking stations.

- First alignment of 24 OT half-layers with cosmic rays.

- Align for Tx (16 DoFs, 2 first and 2 last layers fixed).

- Fix average global translations / rotations / shearings.

- Track selection: ==> 8'000 OT tracks left for alignment.

- Convergence in 2 iterations because not all hits found by pattern recognition in first iteration due to large misalignments.

- Survey measurements only good to $2 \mathrm{~mm}$.

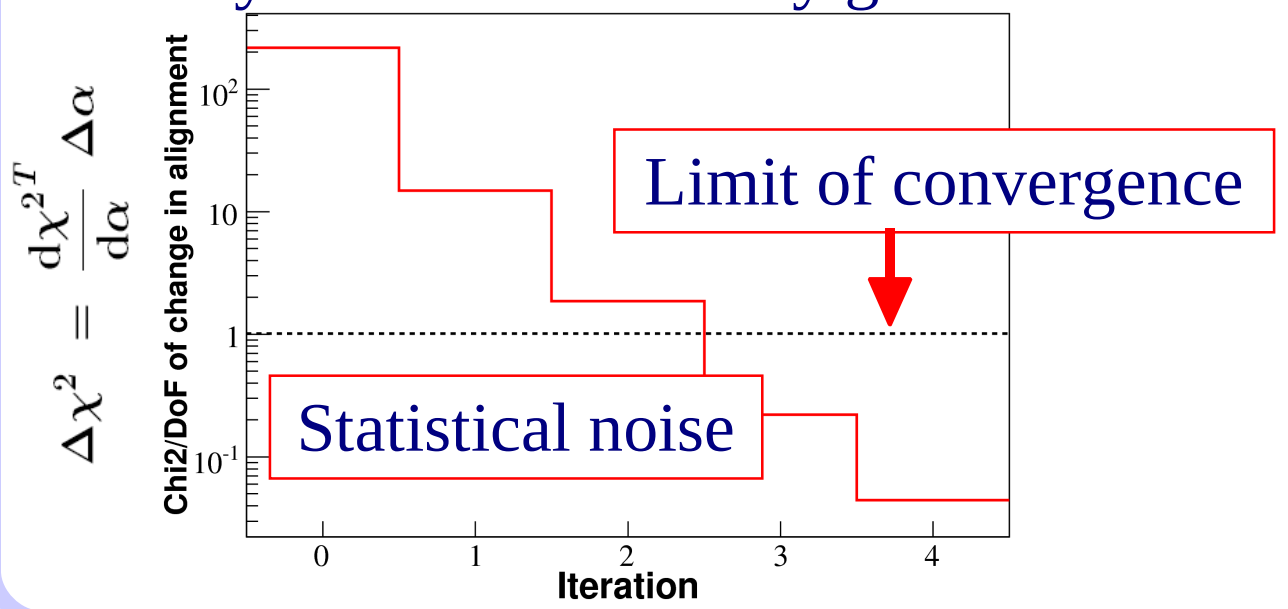

correction to survey

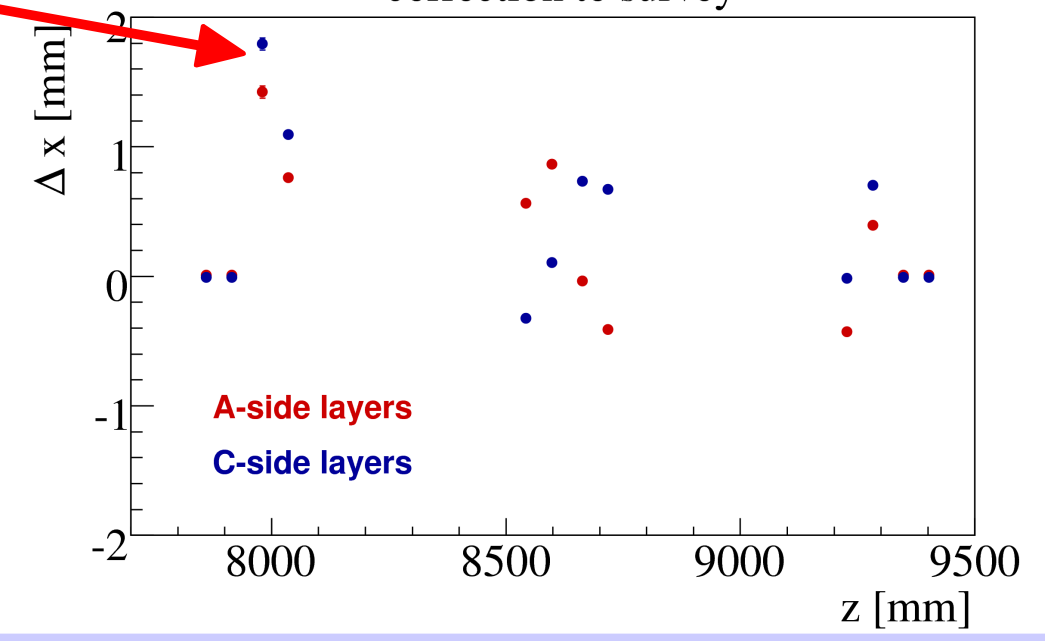

Louis Nicolas - LPHE/EPFL

Software for Detectors@ NSS/IEEE

$11 / 12$

October 21, 2008 
- Alignment framework using all the standard LHCb tracking tool.

- No global track model is needed. We use the Kalman-fitted tracks: same tracks as for physics studies, with full complexity (multiple scattering, $\mathrm{dE} / \mathrm{dx}$ correction, ...).

- We can align any detector element and for any DoF (translations + rotations).

- IT-OT simultaneous alignment has been tested on realistic day-1 misalignment scenarios.

- OT alignment has been performed on real cosmic rays data: procedure works!

- The points to be careful about are:

- Track selection.

- Weak mode removal.

- Alignment in several steps.

- Future plans:

- More studies of the existing cosmic rays and beam - dump data.

- Ready for beam - gas and p - p collisions data. 


\section{END}




\section{BACKUP SLIDES}


- We use a global (closed-form) $\chi^{2}$ minimization:

$$
\frac{\partial \sum_{i} \chi_{i}^{2}}{\partial \alpha}=0 \quad \text { and } \quad \forall_{\text {tracks } i} \frac{\partial \chi_{i}^{2}}{\partial X_{i}}=0
$$

- Compute total derivatives to alignment parameters, by eliminating derivatives to track parameters:

$$
\begin{aligned}
\frac{\mathrm{d} \chi^{2}}{\mathrm{~d} \alpha}= & 2 \sum_{\text {tracks }} \frac{\partial r^{T}}{\partial \alpha} V^{-1} r \quad \frac{\mathrm{d}^{2} \chi^{2}}{\mathrm{~d} \alpha^{2}}=2 \sum_{\text {tracks }} \frac{\partial r^{T}}{\partial \alpha} V^{-1}\left(V-H C I^{T}\right) V^{-1} \frac{\partial r}{\partial \alpha} \\
& \mathrm{V}=\text { measurement covariance matrix. } \\
& \mathrm{H}=\text { Derivatives of residuals to track parameters. } \\
& \mathrm{C}=\text { Track covariance matrix: incomplete in Kalman filter (no correlations). }
\end{aligned}
$$

- Mathematically equivalent to Millepede method: in e.g. V. Blobel, "Software alignment for tracking detectors", NIM A 566 (2006) 5.

- Notable difference in our method: using Kalman track fit.

- Calculation of track covariance matrix $\mathrm{C}$ is not trivial. The maths for this novel calculation are described in arXiv:0810.2241v1. 
- Run 8 iterations over 50'000 beam - residual gas, magnet-off events with $450 \mathrm{GeV}$ protons.

- Misalignment scenario: Reasonable day-1 scenario where IT and OT layers have been misaligned at the level of Tx ( $\pm 300 \mu \mathrm{m})$, Ry and Rz ( $\pm 3 \mathrm{mrad}$ )

- Standalone alignment with T-tracks (IT + OT) for IT and OT simultaneously.

- Important points for the convergence of the alignment algorithm:

- Track selection: ==> 4.9 tracks $(\mathrm{IT}+\mathrm{OT})$ / event left for alignment.

- Select event with low occupancy to reduce ghost rate.

- Use evolving $\chi^{2}$ cut to remove ghost tracks and interactions.

- Drift time off in OT in first 4 iterations, turned on after 4 iterations.

- Convergence more stable without L/R ambiguity resolution.

- But worse precision (1 mm vs $200 \mu \mathrm{m}$ ).

- Strategy: get close to minimum, then use power of drift time information. 
- Total sum of track $\chi^{2}$ vs iterations:

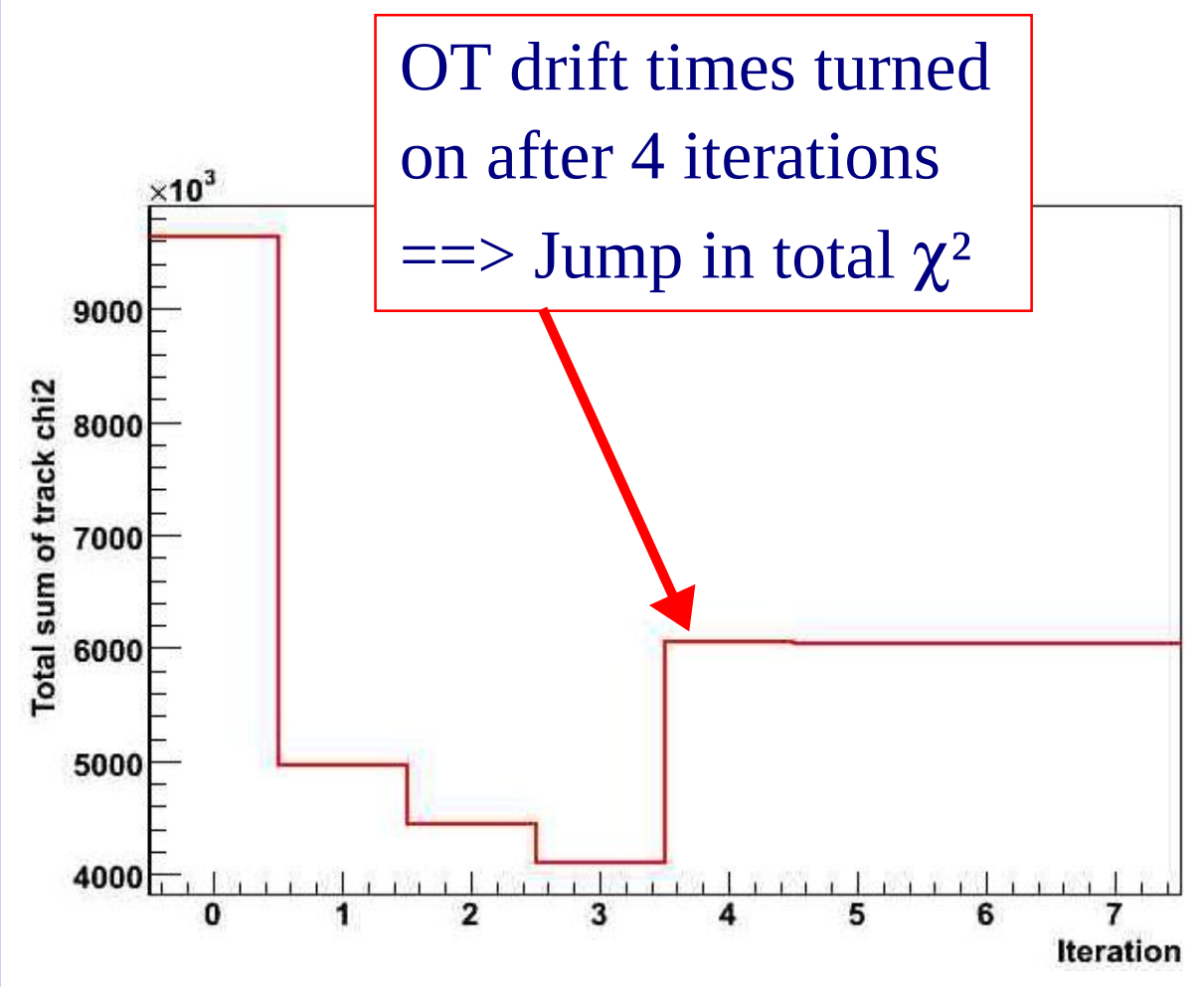

- Input - output Tx for all IT/OT layers:

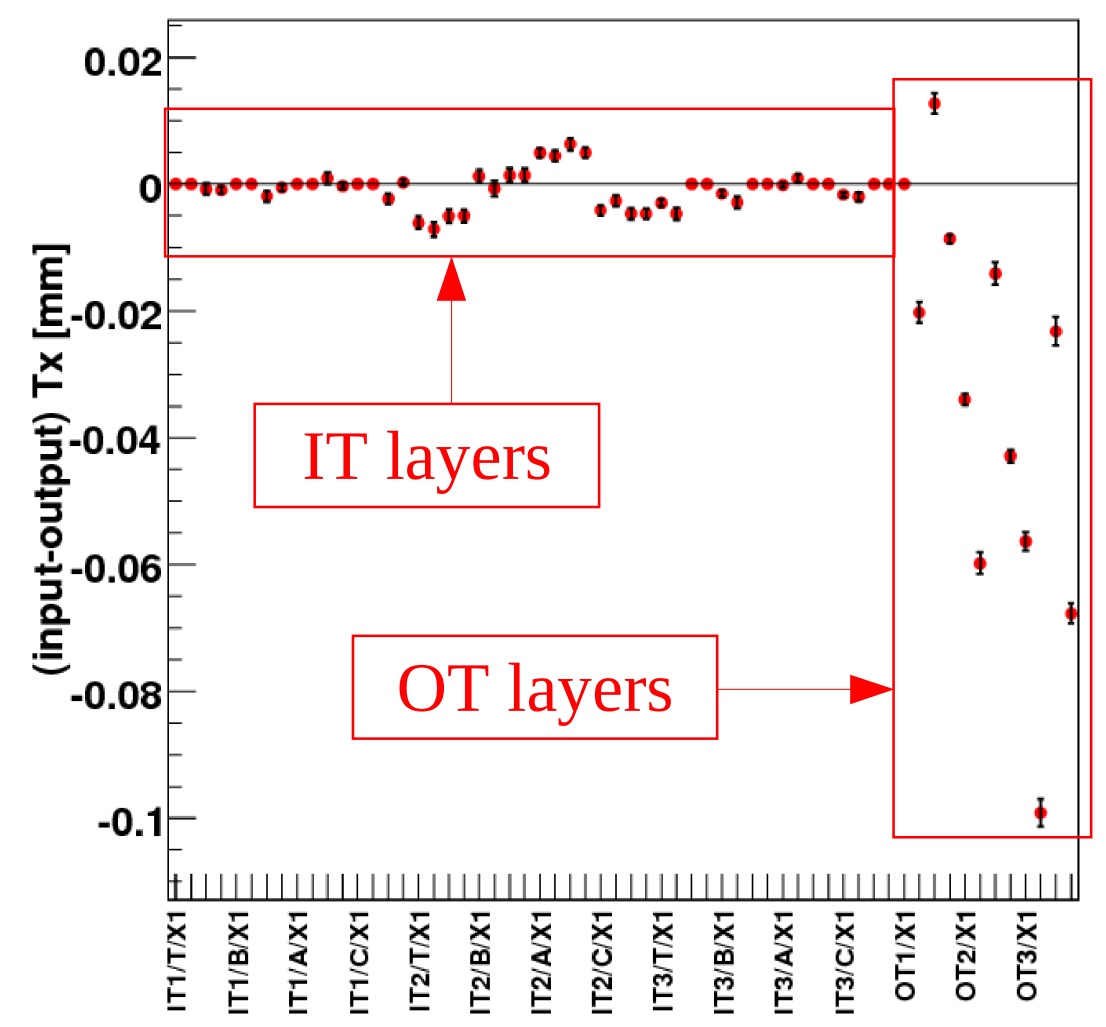

- Looking at $\chi^{2}$ convergence: alignment converged in 4-5 iterations.
- IT layers aligned in Tx within $10 \mu \mathrm{m}$.

- OT layers aligned in Tx within $100 \mu \mathrm{m}$ (without drift times: to $\pm 180 \mu \mathrm{m}$ ). 
Alignment algorithm has converged, but where are we from the physics point of view?

- Run over 65'500 inclusive J/ $\psi$.

- Loose $\mathrm{J} / \psi$ selection

$$
==>31 ' 918 \mathrm{~J} / \psi \text { candidates. }
$$

- Refit of tracks from J/ $\psi$ with 3 geometries:

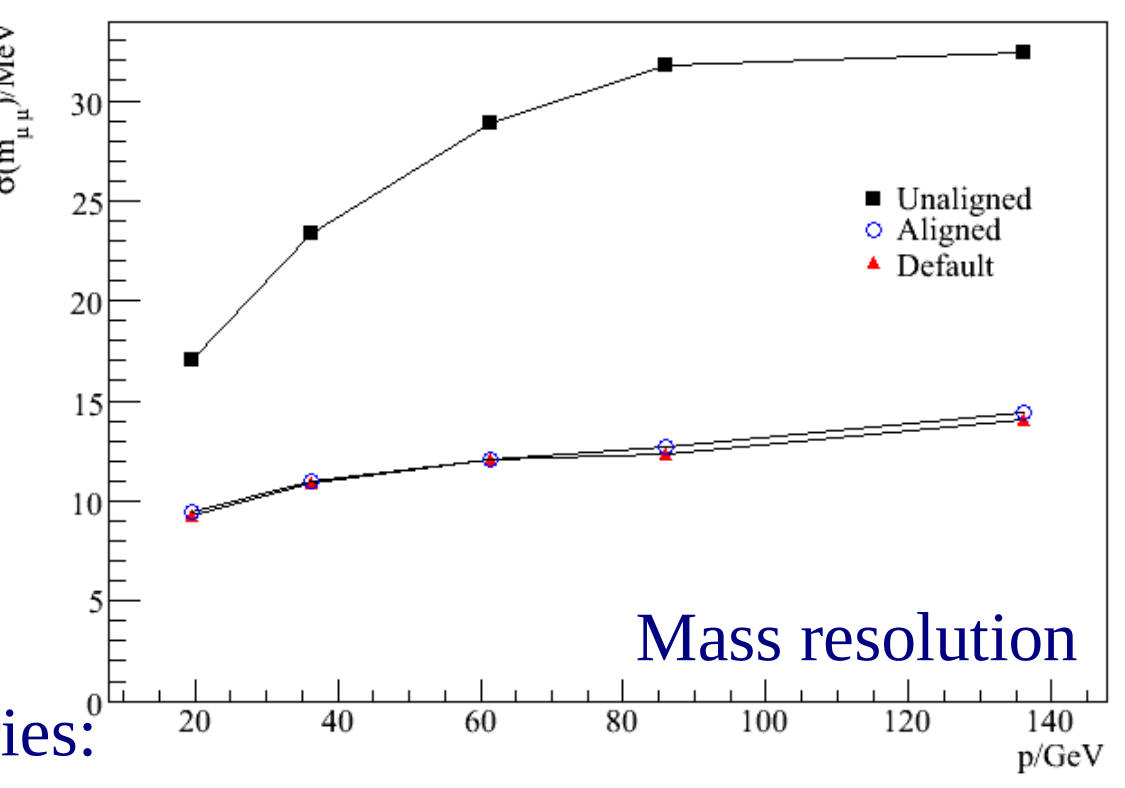

- Unaligned (before alignment job).

- Aligned (after alignment job).

- Default (ideal geometry).

- Look at:

- Dimuon mass resolution:

$$
\text { M(rec) - M(true } \mu \mu \text { ). }
$$

- Track $\chi^{2} /$ dof.

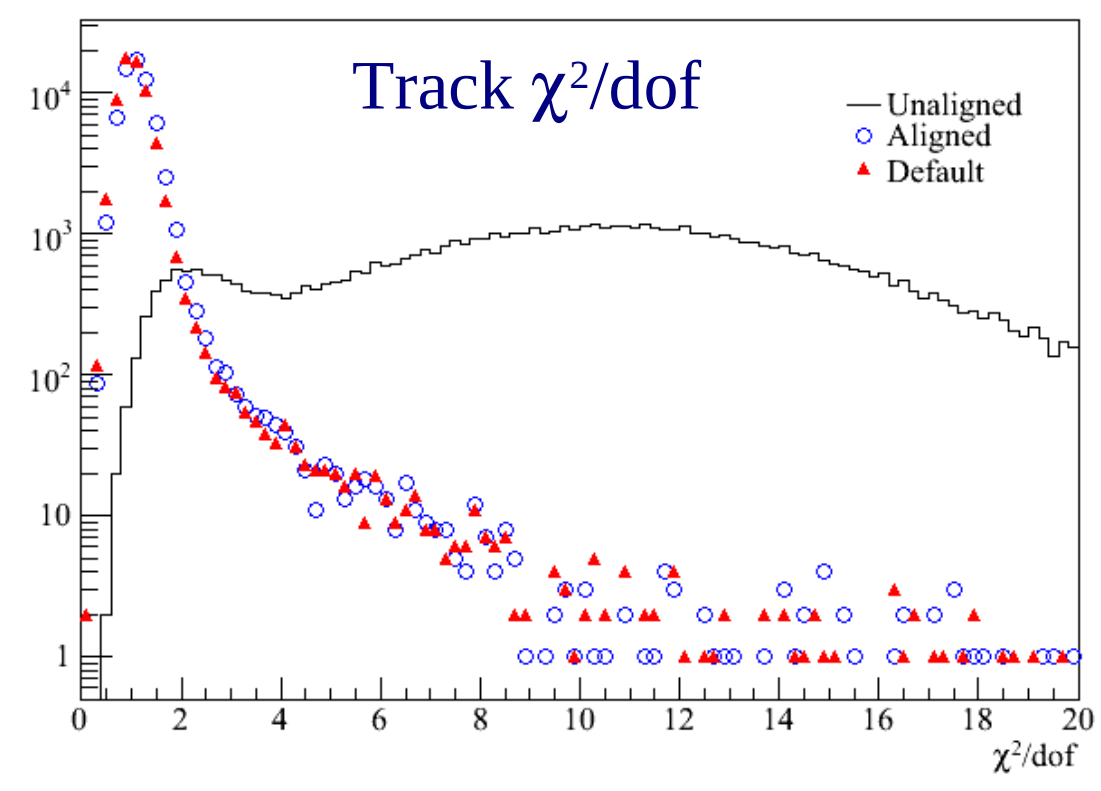


- Distance between TT hits and extrapolated VeLo segments for TT layers:

Before alignment

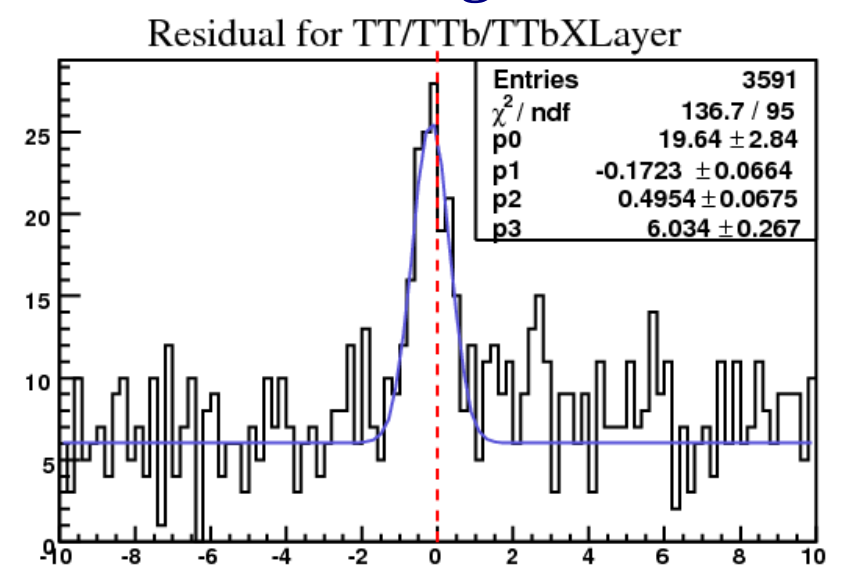

Residual for TT/TTa/TTaXLayer

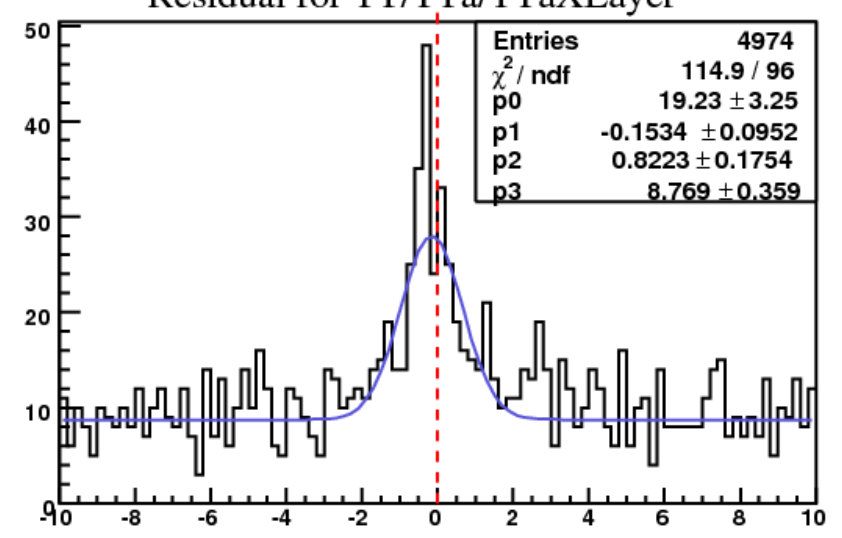

After alignment

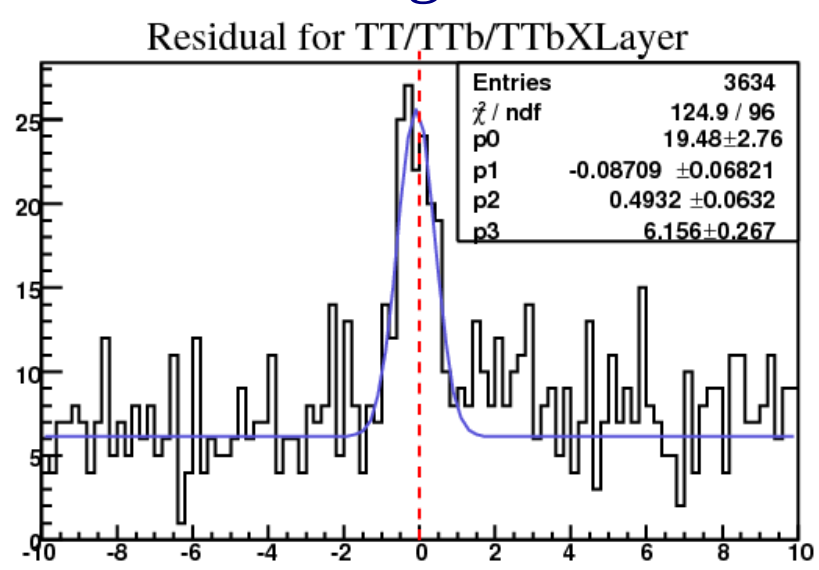

Residual for TT/TTa/TTaXLayer

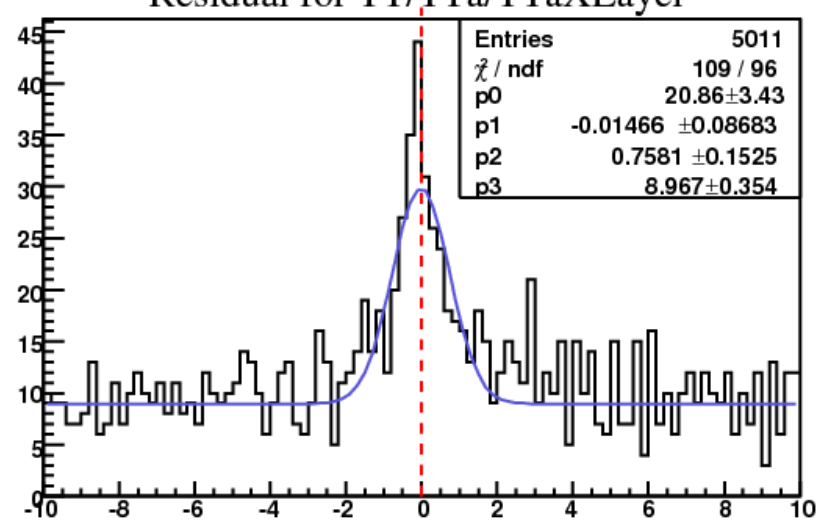

- Hits within $\sim 300 \mu \mathrm{m}$ of expected, with a resolution of $\sim 500 \mu \mathrm{m}$.

- Peak goes back to the right place after alignment. 
- Distance between IT hits and extrapolated VeLo segments for IT layers.

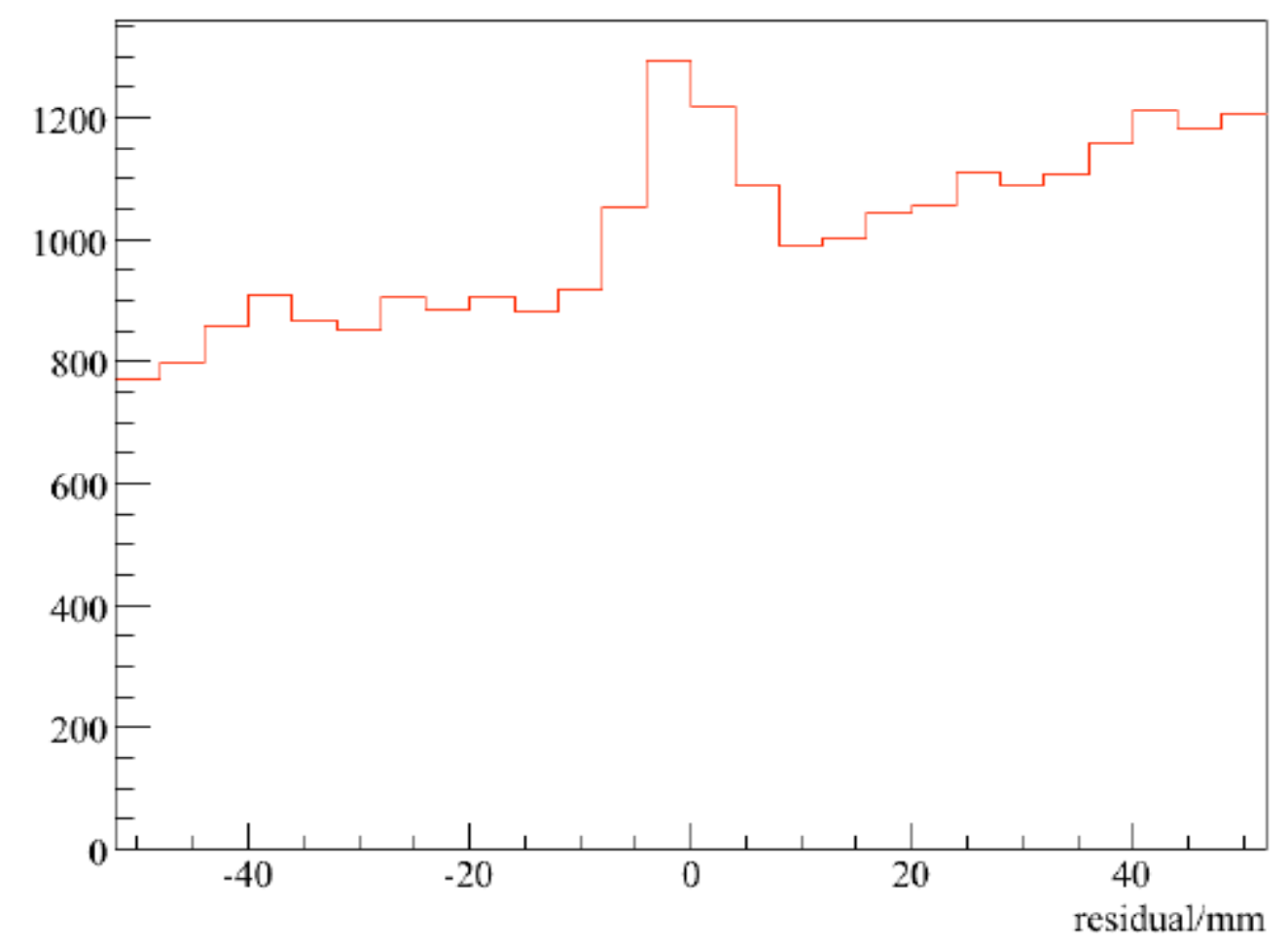

- Looks like a resolution of $\sim 3 \mathrm{~mm}$.

- But very long (several meters) extrapolation. 
- Take any pair of hits in T1 and T3, draw a line.

- Plot distance between line and all the hits in T2.

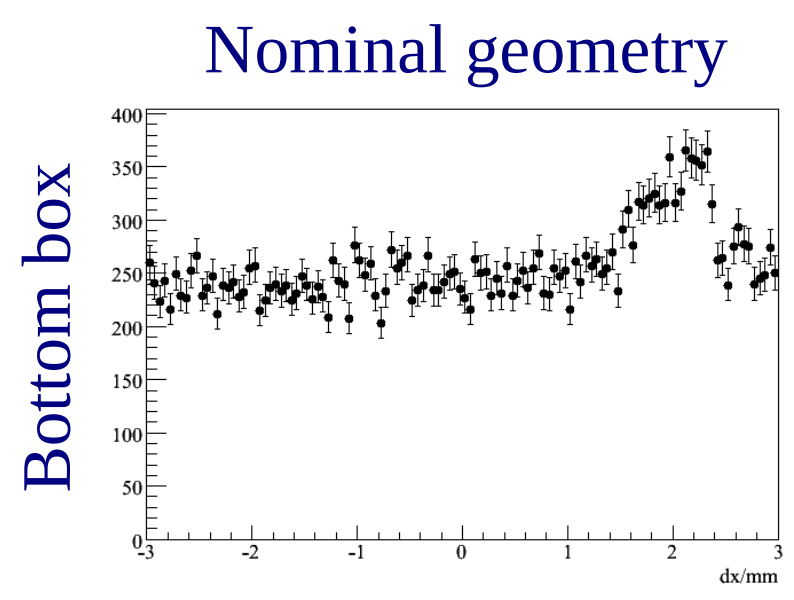

Survey measurements
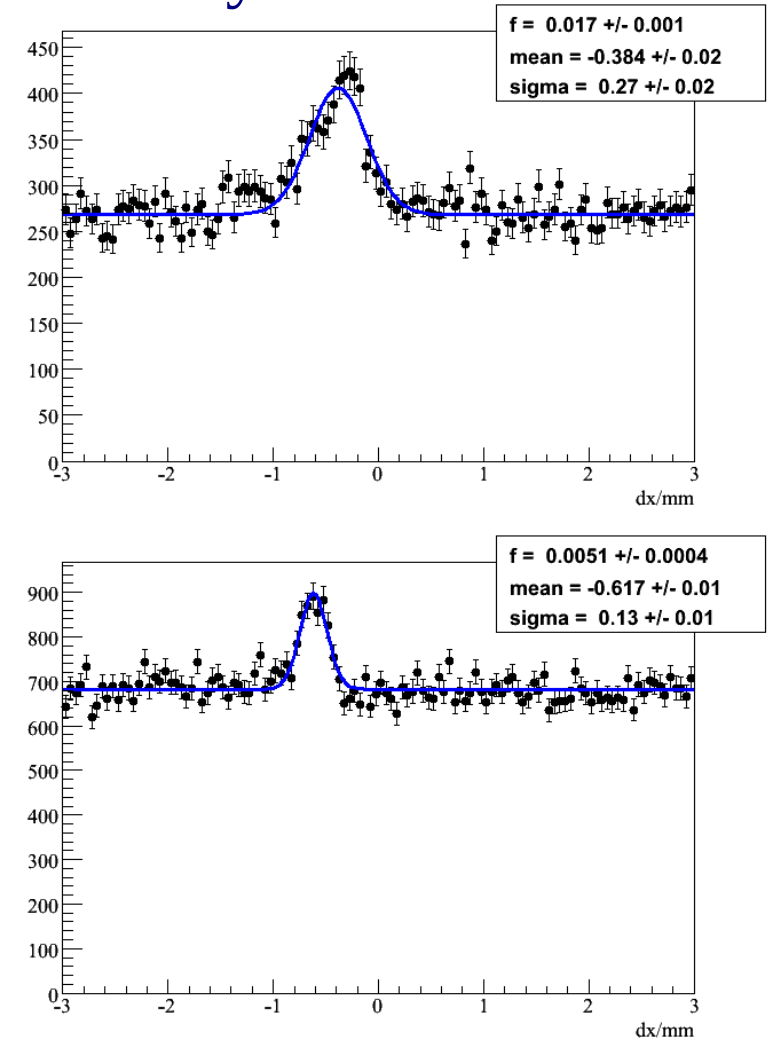

Additional corrections
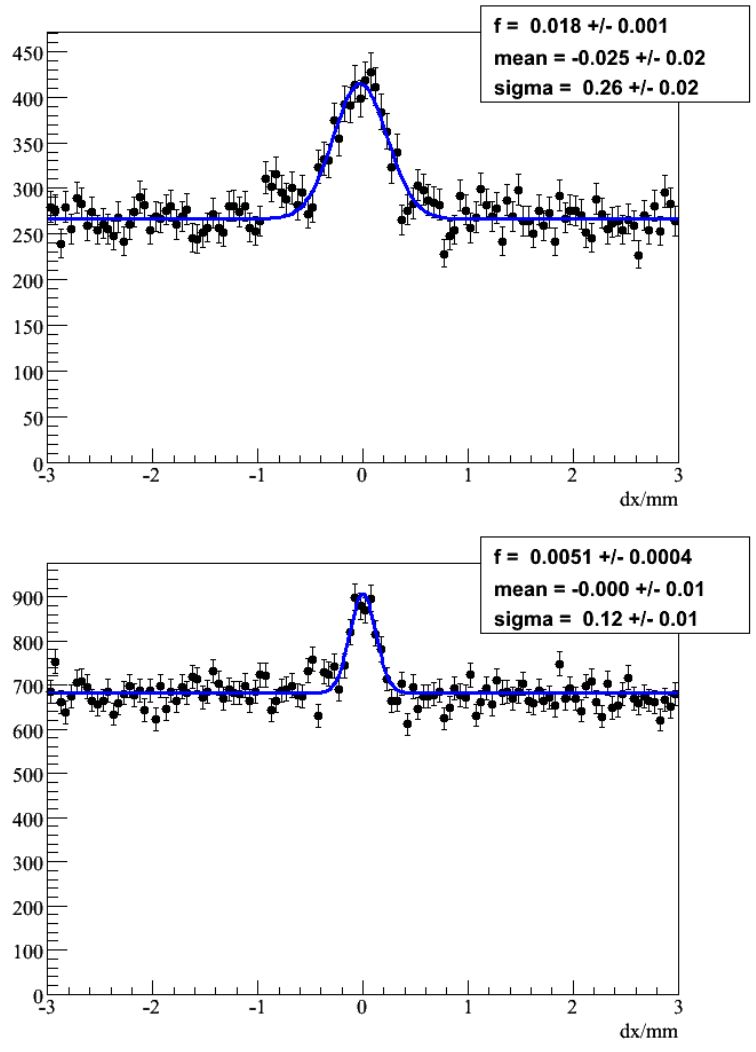

- Occupancy 10 times larger than nominal running conditions. $==>$ Very hard to find tracks in IT. 